\title{
Method and System for Human Action Detections with Acceleration Sensors for the Proposed Rescue System for Disabled and Elderly Persons Who Need a Help in Evacuation from Disaster Area
}

\author{
Kohei Arai ${ }^{1}$ \\ Graduate School of Science and Engineering \\ Saga University \\ Saga City, Japan
}

\begin{abstract}
Method and system for human action detections with acceleration sensors for the proposed rescue system for disabled and elderly persons who need a help in evacuation from disaster areas is proposed. Not only vital signs, blood pressure, heart beat pulse rate, body temperature, bless and consciousness, but also, the location and attitude of the persons have to be monitored for the proposed rescue system. The attitude can be measured with acceleration sensors. In particular, it is better to discriminate the attitudes, sitting, standing up, and lying down. Also, action speed has to be detected. Experimental results show that these attitude monitoring can be done with acceleration sensors.
\end{abstract}

Keywords—vital sign; heart beat puls ratee; body temperature; blood pressure; blesses, consciousnes; seonsor network

\section{INTRODUCTION}

Handicapped, disabled, diseased, elderly persons as well as peoples who need help in their ordinary life are facing too dangerous situation in event of evacuation when disaster occurs. In order to mitigate victims, evacuation system has to be created. Authors proposed such evacuation system as a prototype system already [1]-[4].

The system needs information of victims' locations, physical and psychological status as well as their attitudes. Authors proposed sensor network system which consist GPS receiver, attitude sensor, physical health monitoring sensors which allows wearable body temperature, heart beat pulse rates; bless monitoring together with blood pressure monitoring [5]-[7]. Also the number of steps, calorie consumptions is available to monitor. Because it is difficult to monitor the blood pressure with wearable sensors, it is done by using the number of steps and body temperature. In addition to these, psychological status is highly required for vital sign monitoring (consciousness monitoring). By using EEG sensors, it is possible to monitor psychological status in the wearable sensor. These are components of the proposed physical health and psychological monitoring system.

Method and system for human action detections with acceleration sensors for the proposed rescue system for disabled and elderly persons who need a help in evacuation from disaster area is proposed. Experimental results show that human actions can be estimated with acceleration sensors.

Section 2 describes the proposed acceleration sensor system followed by experiment method and results. Then conclusion is described together with some discussions..

\section{Proposed SENSOR Network SySteM}

\section{A. Acceleration Sensor Used}

Figure 1 shows outlook of the acceleration sensor used in the proposed rescue system. It is the Small Sized Wireless Hybrid Sensor WAA-010

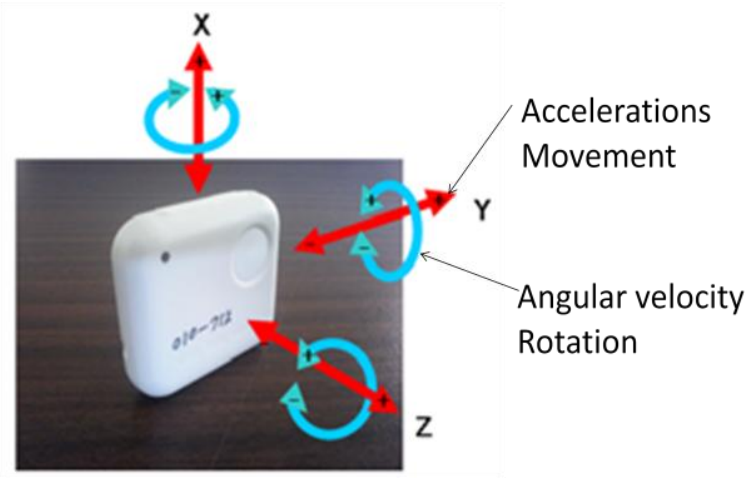

Fig. 1. Outlook of the Small Sized Wireless Hybrid Sensor WAA-010.

It allows measurements of movements in $\mathrm{x}, \mathrm{y}$, and $\mathrm{z}$ directions and roll, pitch and yaw rotations. WAA-010 is a multi sensor which allows measurements of attitude, angular velocity (gyro), Earth magnet in three axes. Also WAA-010 has communication capability to PC through Bluetooth communication links.

\section{B. Acceleration Data Acquisition}

Figure 2 (a) shows the toggle of the Bluetooth while Figure 2 (b) shows installation window of Bluetooth on PC display. 


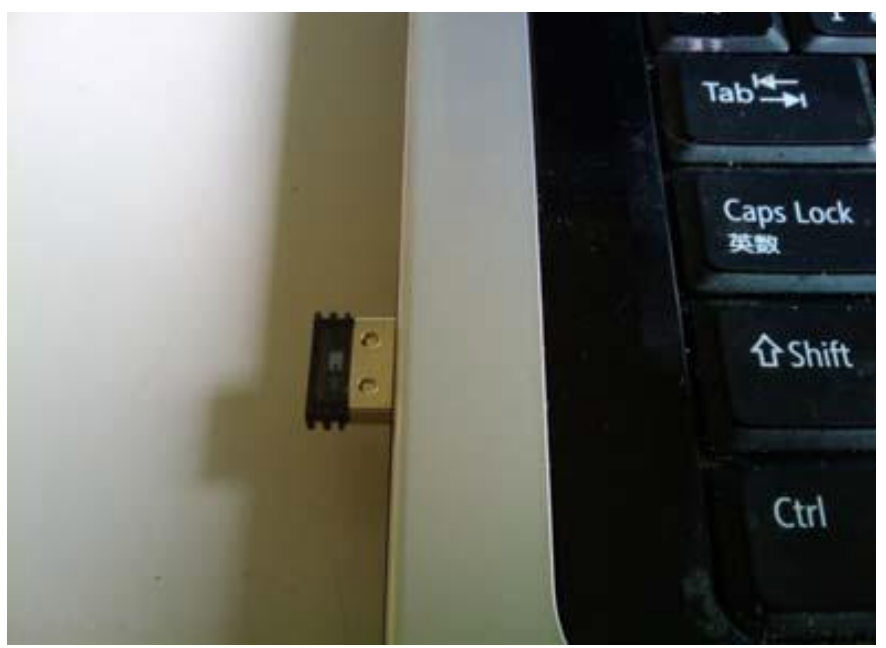

(a)Toggle of the Bluetooth

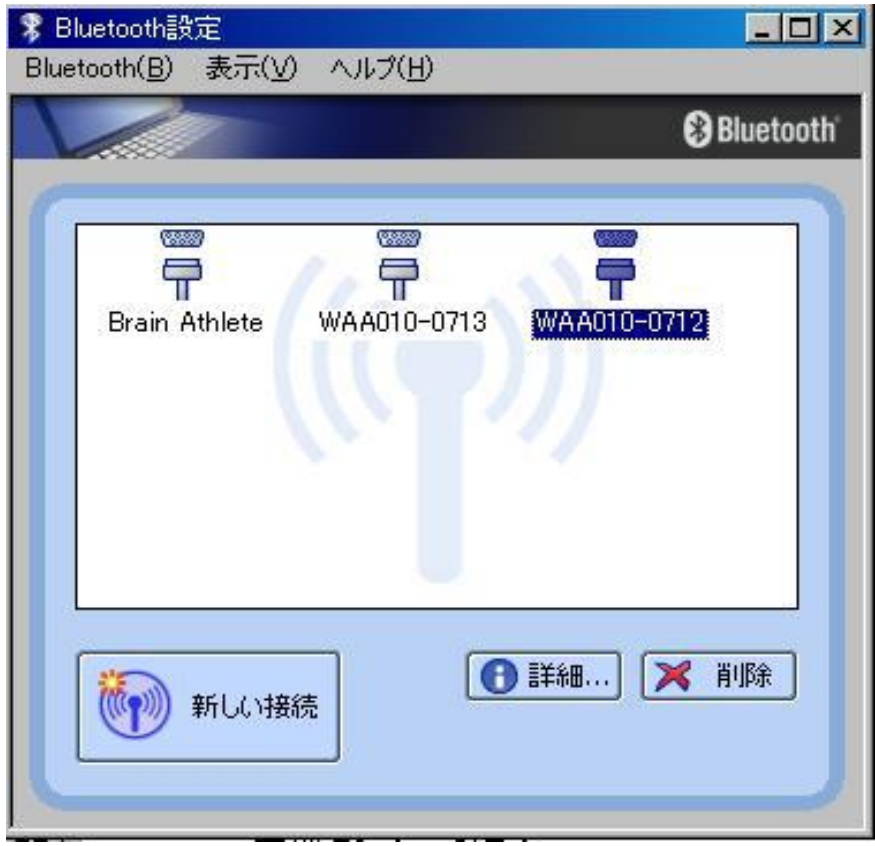

(b) Installation window of Bluetooth on PC display.

Fig. 2. Toggle of the Bluetooth and installation window of Bluetooth on PC display.

After the installation of acceleration sensor driver, communication pot assignments, batteries of the three axis of acceleration, angular velocity as well as Earth magnet-meter can be monitored through PC screen which is shown in Figure 3.

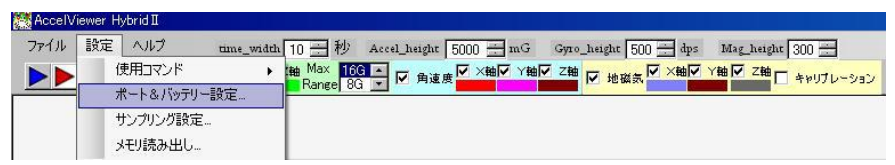

(a)Port asignment

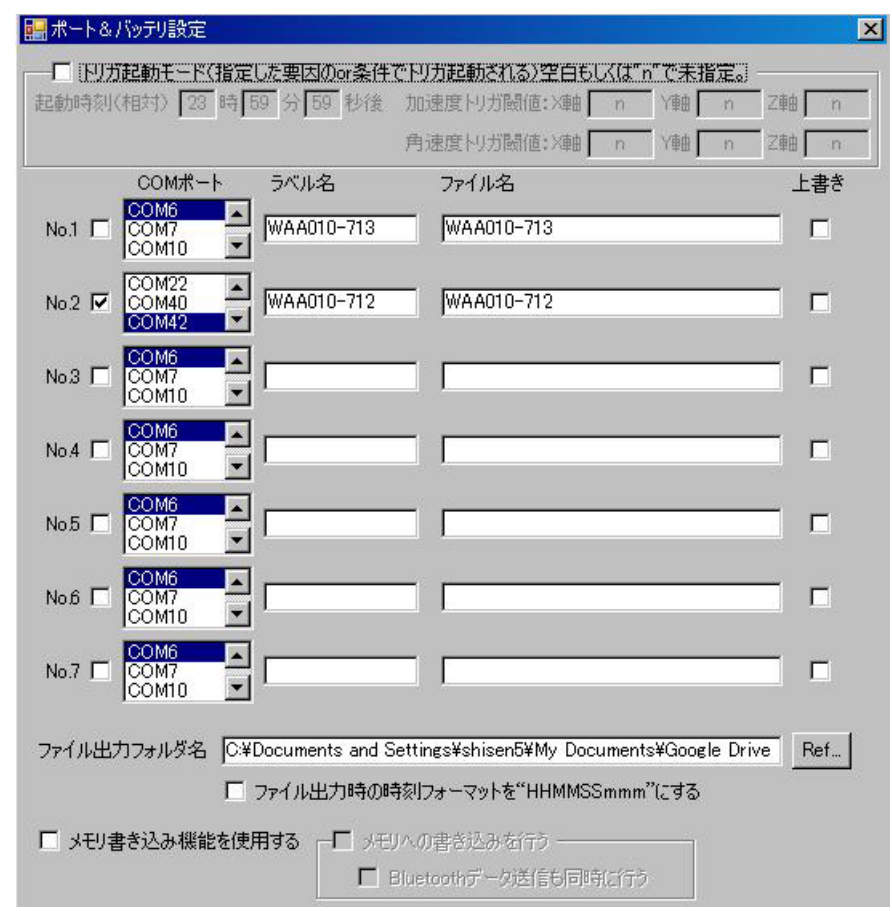

(b)Battery monitor

Fig. 3. Communication port assignment and battery monitor

\section{EXPERIMENTS}

\section{A. Examples of Acceleration and Angular Velocity Data}

Figure 4 (a) shows an example of acceleration sensor data with the maximum range of $5000 \mathrm{mG}$ while Figure 4 (b) shows and example of measured acceleration sensor data with the maximum range of $2000 \mathrm{mG}$. As shown in Figure 4, the maximum range has to be adjusted for the acceleration of the target objects. Meanwhile, Figure 5 (a) and (b) shows an example of measured angular velocity with maximum of 500 dps and 200 dps, respectively. These examples are obtained through the experiments of which the acceleration sensor are held by hands. Therefore, these performances are just for the acceleration sensor only.

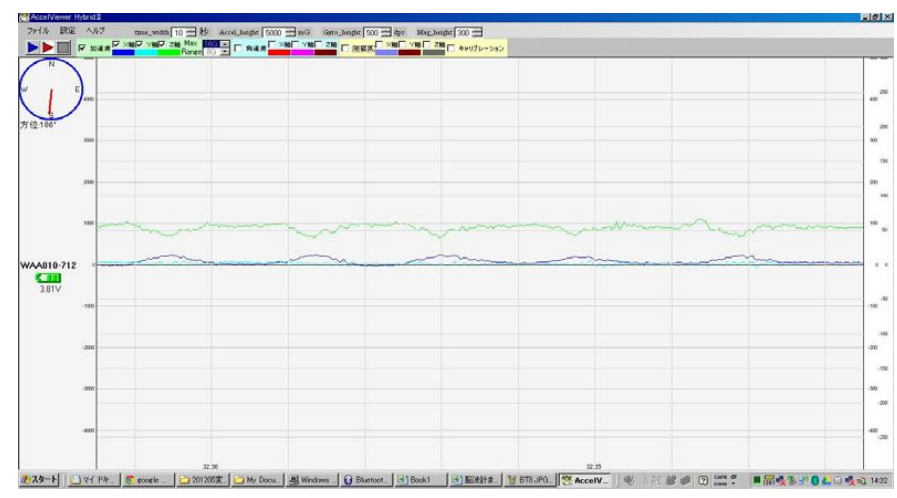

(a) $5000 \mathrm{mG}$ 


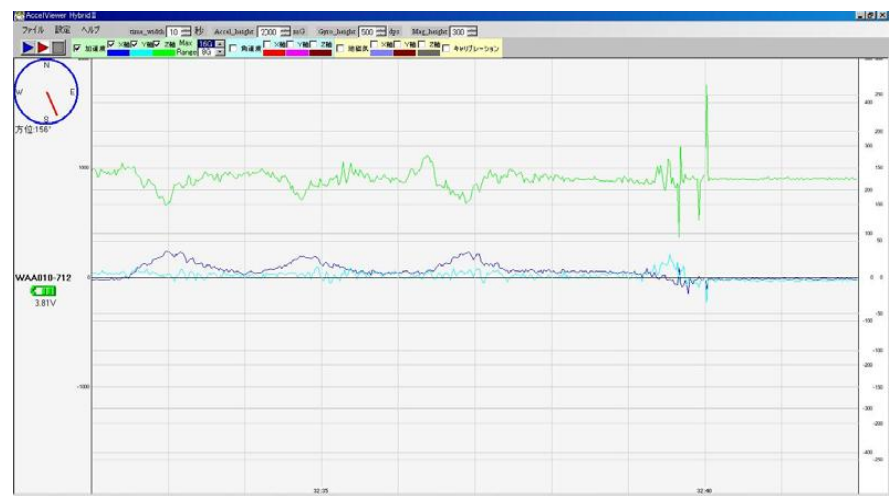

(b) $2000 \mathrm{mG}$

Fig. 4. Examples of acceleration sensor data

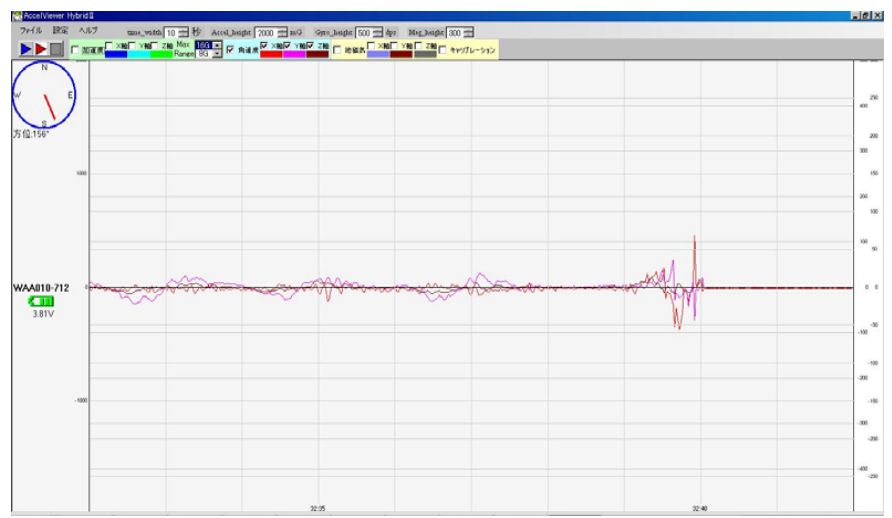

(a)500dps

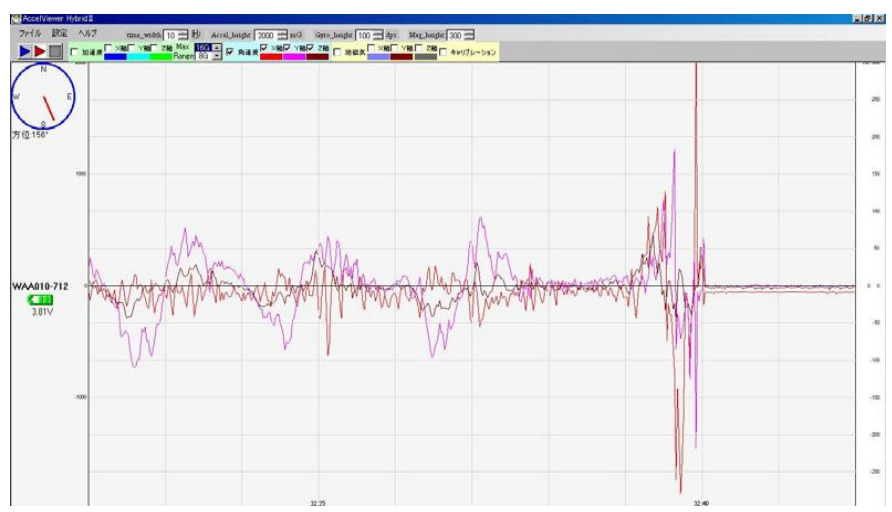

(b)200dps

Fig. 5. Examples of angular velocity measured

Meanwhile, example of acceleration sensor data $(200 \mathrm{mG}$ of maximum range) for the motion in $\mathrm{Z}$ direction is shown in Figure 6 (a).

On the other hand, Figure 6 (b) and (c) shows examples of acceleration sensor data for the motion in $\mathrm{Y}$ and $\mathrm{X}$ directions, respectively.

Figure 7 (a), (b), and (c) shows examples of angular velocity data (500 dps of maximum range) for the rotations in $\mathrm{Z}, \mathrm{Y}, \mathrm{X}$ directions, roll, pitch and yaw angles, respectively.

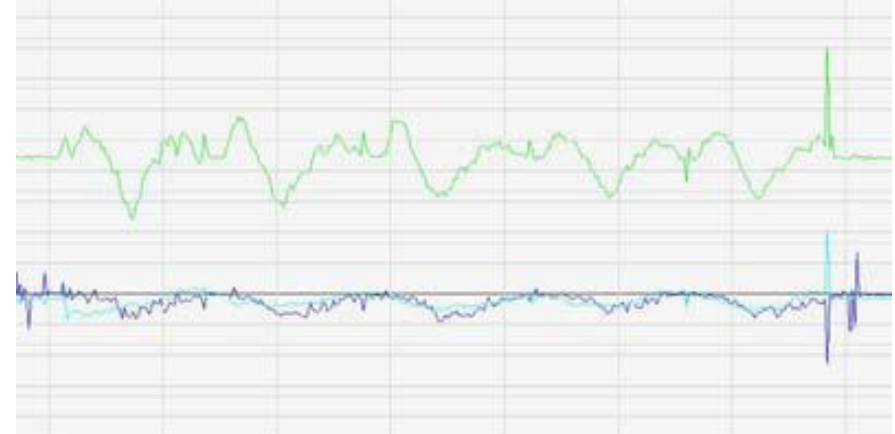

(a) $\mathrm{Z}$ direction

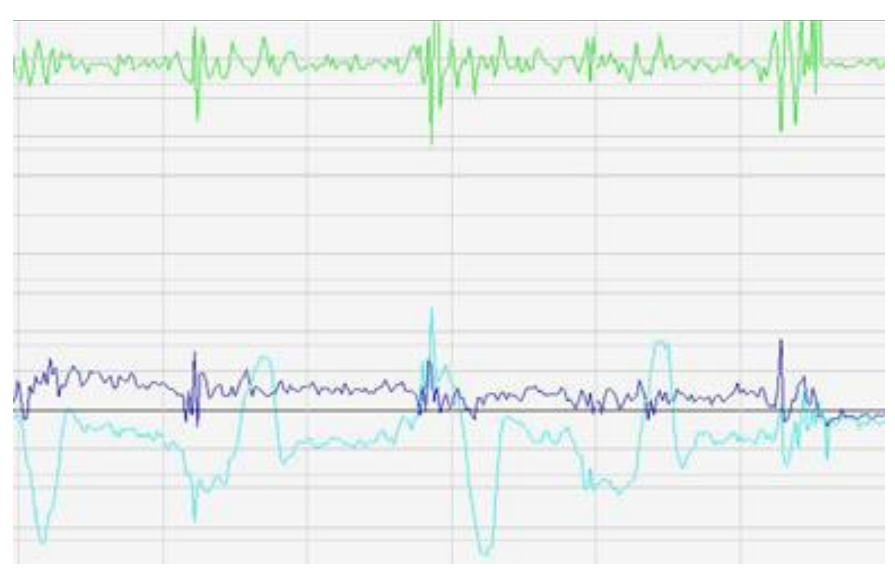

(b) Y direction

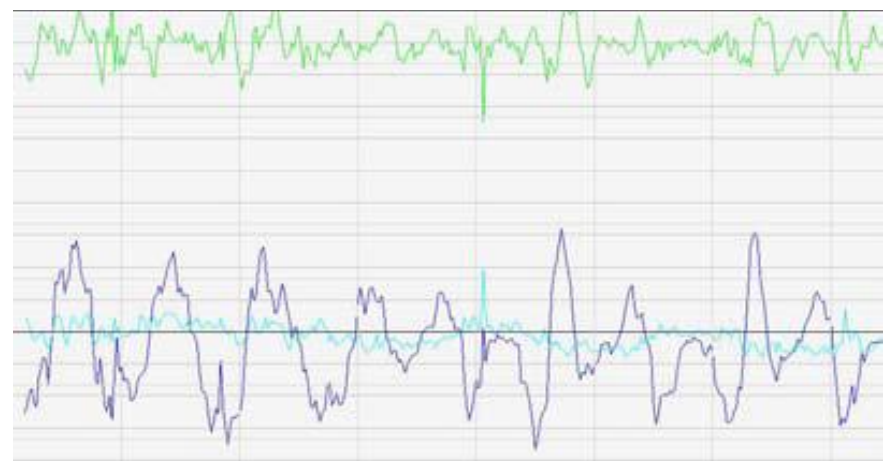

(c) $\mathrm{X}$ direction

Fig. 6. Examples of acceleration sensor data for the motions in $\mathrm{X}, \mathrm{Y}$, and $\mathrm{Z}$ directions

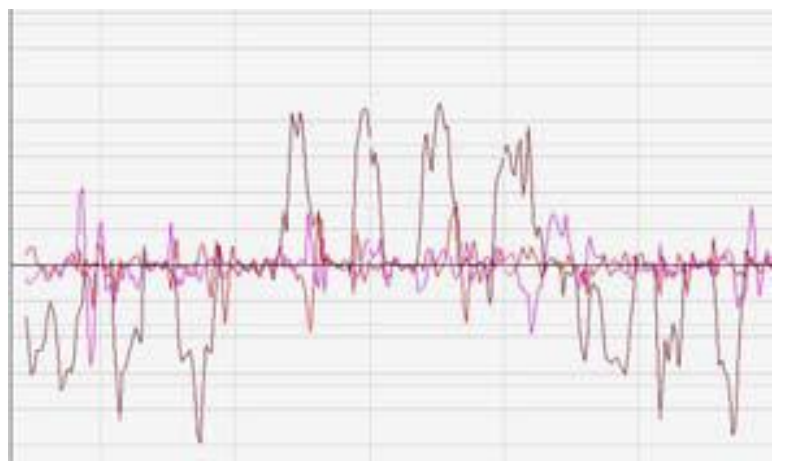

(a)Rotation in $\mathrm{Z}$ direction 


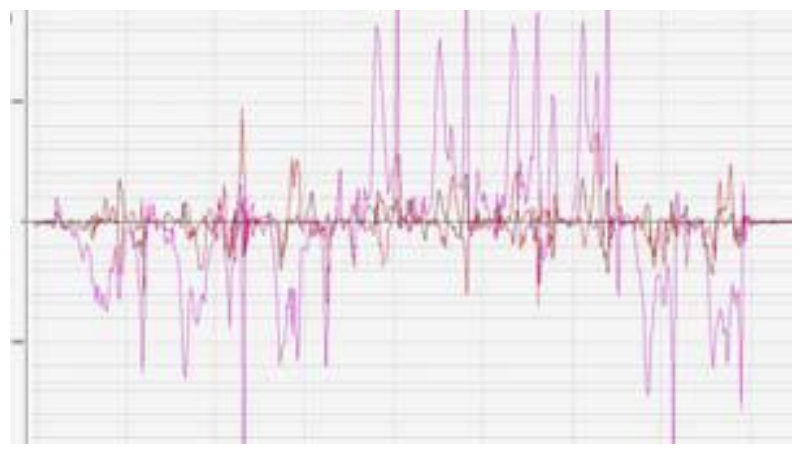

(b)Rotation in Y direction

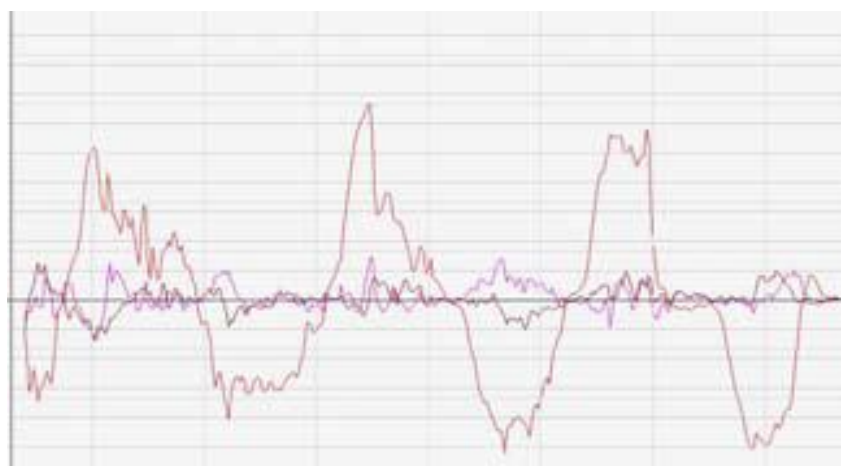

(c)Rotation in $\mathrm{X}$ direction

Fig. 7. Examples of angular velocity data

\section{B. Examples of Acceleration and Angular Velocity Data Attached to the Wearing Glass End}

Acceleration sensor is then attached to the wearing glass end as is shown in Figure 8. Not only the acceleration sensor but also body temperature, heart beat pulse rate sensors as well as EEG sensor head are attached to the glass end together with battery and Bluetooth communicator. At the forehead, EEG sensor head is attached with spring wire extended from the center of the glass. Therefore most of vital signs can be measured with the glass.

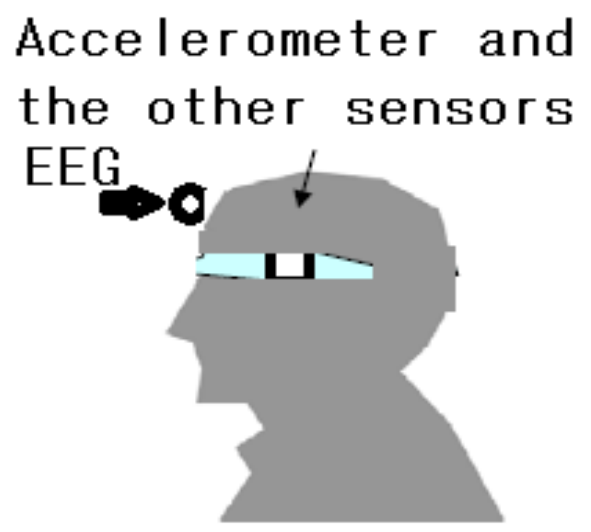

Fig. 8. Location of the acceleration sensor (at the glass end)

Figure 9 shows examples of acceleration sensor and angular velocity data when the user lie down and stand up slowly. Meanwhile, Figure 10 shows examples of those when the user sit down on a chair slowly.

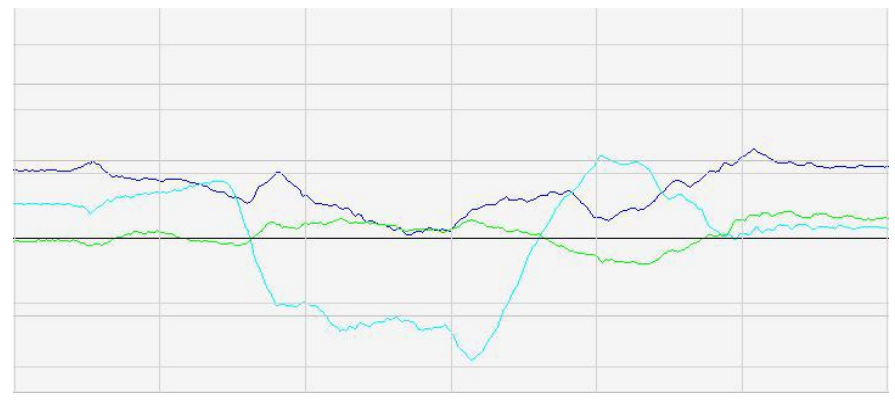

(a)Acceleration (X: Dark Blue, Y: Blue (Effective), Z: Green)

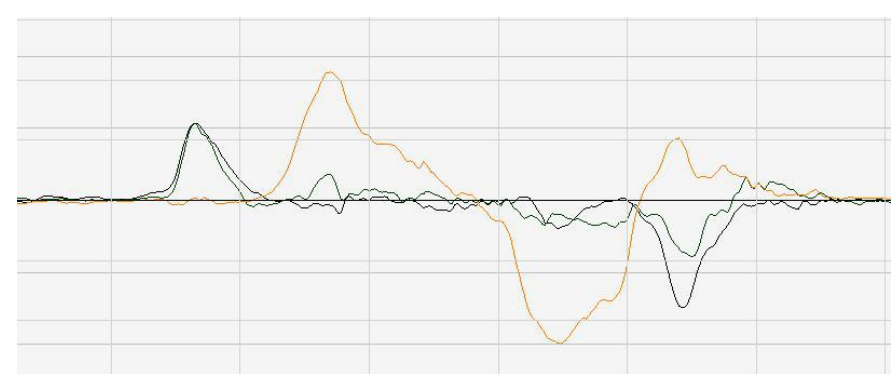

(b)Angular velocity (X: Black, Y: Green, Z: Red (Effective))

Fig. 9. Examples of acceleration sensor and angular velocity data when the user laid down and stand up slowly.

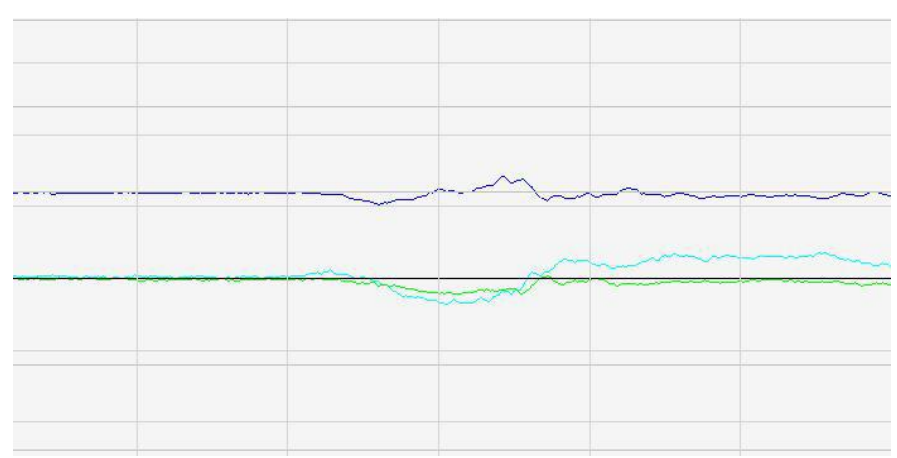

(a)Acceleration (X: Dark Blue, Y: Blue (Effective), Z: Green)

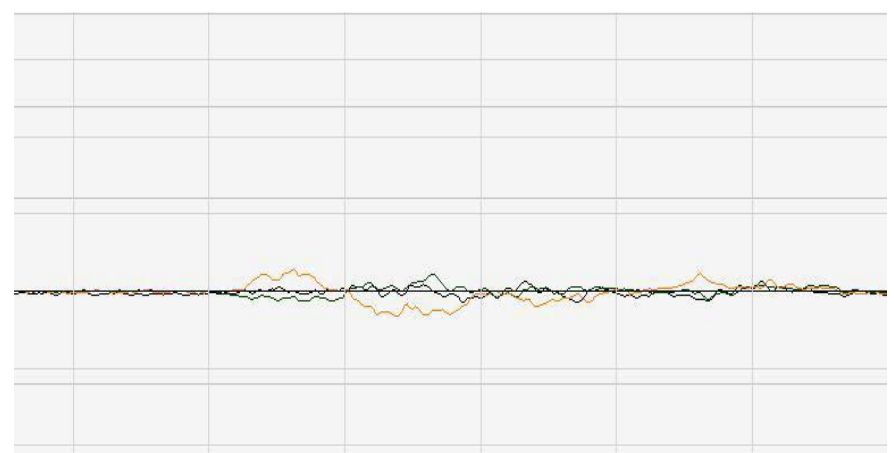

(b)Angular velocity (X: Black, Y: Green, Z: Red (Effective))

Fig. 10. Examples of acceleration sensor and angular velocity data when the user sit down on chair slowly.

Figure 11 shows examples of acceleration sensor and angular velocity data when the user lie down and stand up quickly. Meanwhile, Figure 12 shows examples of those when the user sit down on a chair quickly. On the other hand, Figure 13 (a) shows Y axis data when the user lies down and stand up 
slowly while Figure 13 (b) shows $\mathrm{X}$ axis data when the user sit down on the chair slowly .

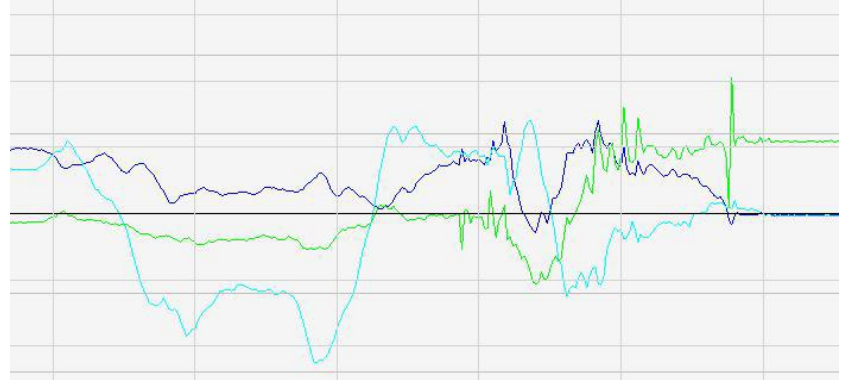

(a)Acceleration (X: Dark Blue, Y: Blue (Effective), Z: Green)

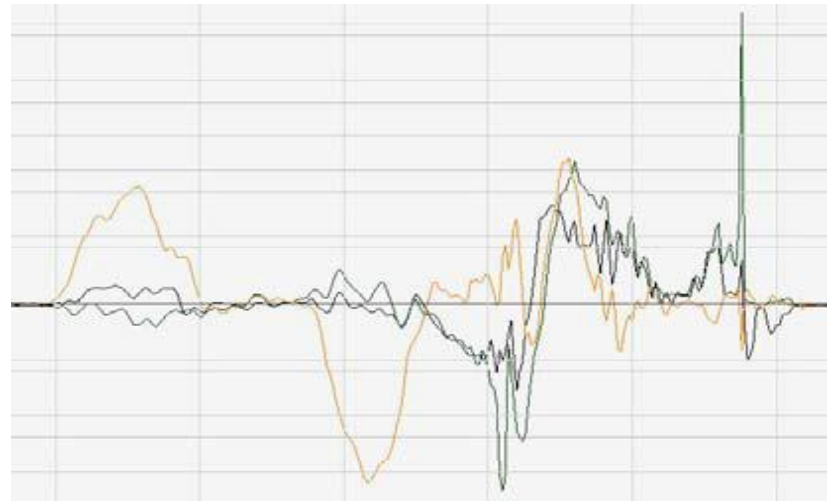

(b)Angular velocity (X: Black, Y: Green, Z: Red (Effective))

Fig. 11. Examples of acceleration sensor and angular velocity data when the user lie down and the stand up quickly.

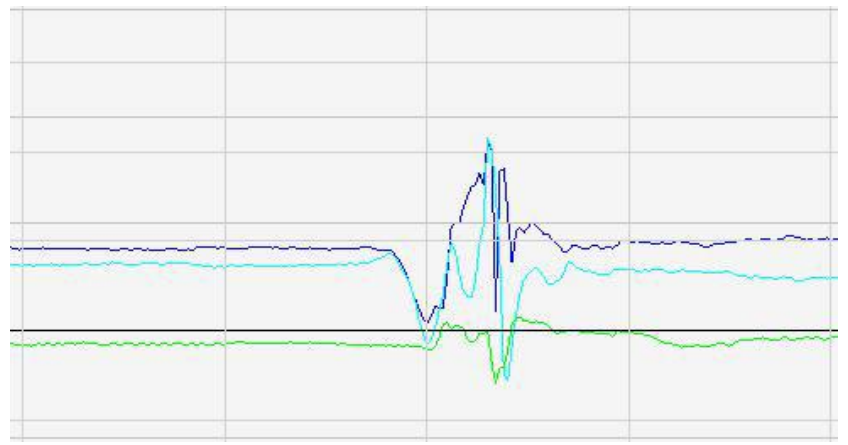

(a)Acceleration (X: Dark Blue, Y: Blue (Effective), Z: Green)

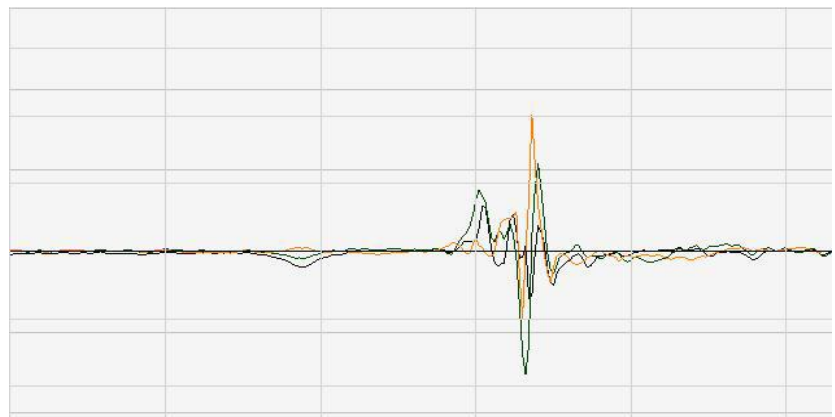

(b)Angular velocity (X: Black, Y: Green, Z: Red (Effective))

Fig. 12. Examples of acceleration sensor and angular velocity data when the user sit down on chair quickly.
Furthermore, Figure 13 (c) shows $\mathrm{Y}$ axis data when the user lies down and the stand up quickly while Figure 13 (d) shows $\mathrm{X}$ axis data when the user sit on the chair quickly.

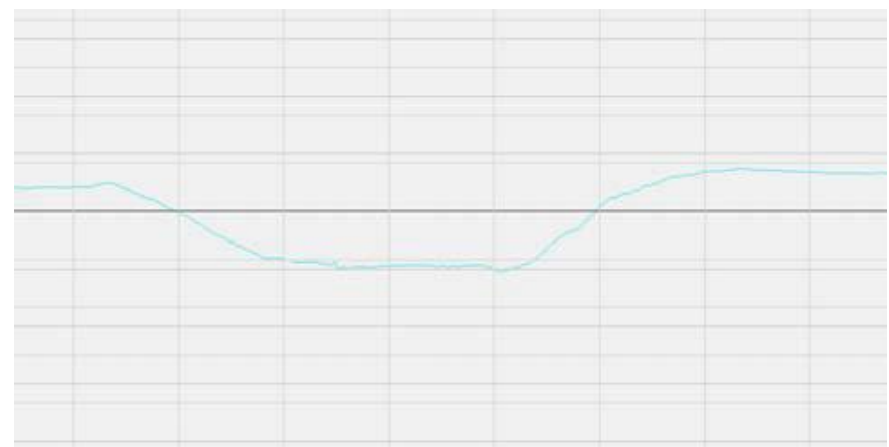

(a) Y axis data when the user lies down and stand up slowly

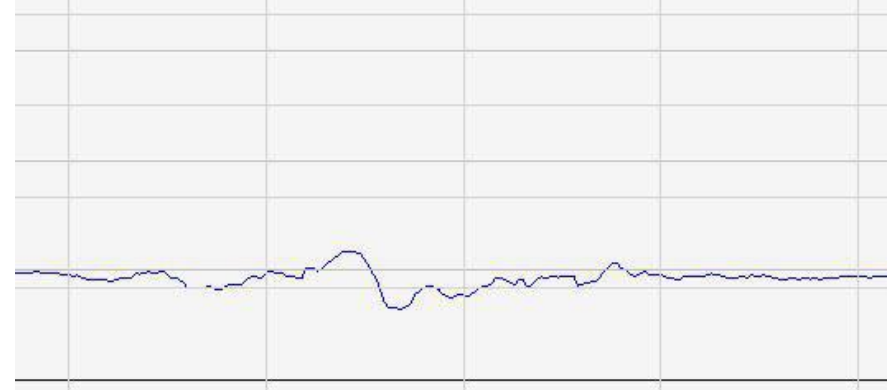

(b) $\mathrm{X}$ axis data when the user sit down on the chair slowly

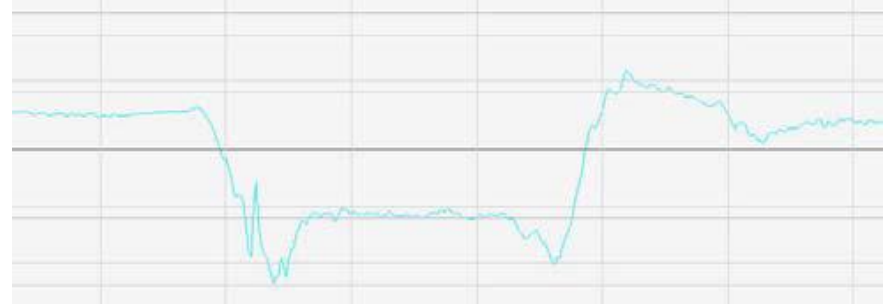

(c) Y axis data when the user lies down and the stand up quickly

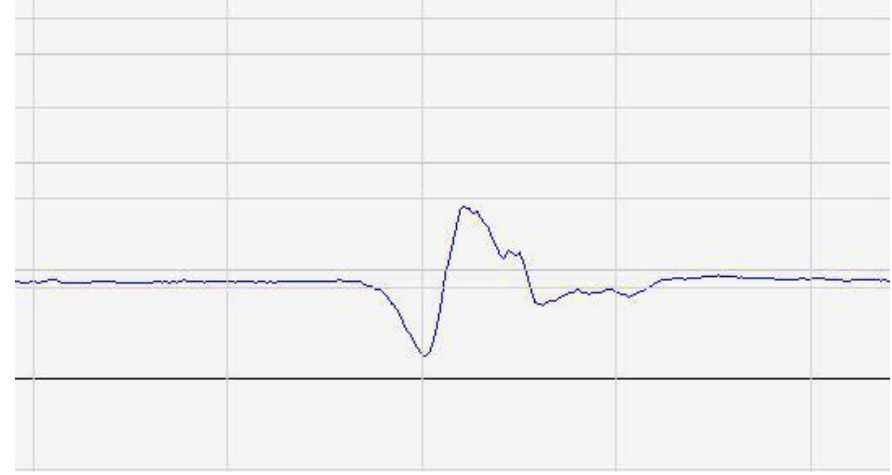

(d) X axis data when the user sit on the chair quickly

Fig. 13. Specific axis data for the action of lie down and then stand up as well as sit down on the chair, slowly and quickly 


\section{Validation of Acceleration and Angular Velocity Data}

Three axes of acceleration sensor and angular velocity sensor data are validated for the actions "Lie down and then stand up slowly", "Sit down on the chair slowly", "Lie down and then stand up quickly", "Sit down on the chair quickly". The results are shown in Figure 14. Through these validations, it is found that the most effective signal for detection of lie down and then stand up is acceleration in $\mathrm{Y}$ axis while that for detecting of sit down action is angular velocity sensor data in $\mathrm{Z}$ axis as are shown in Table 1 .

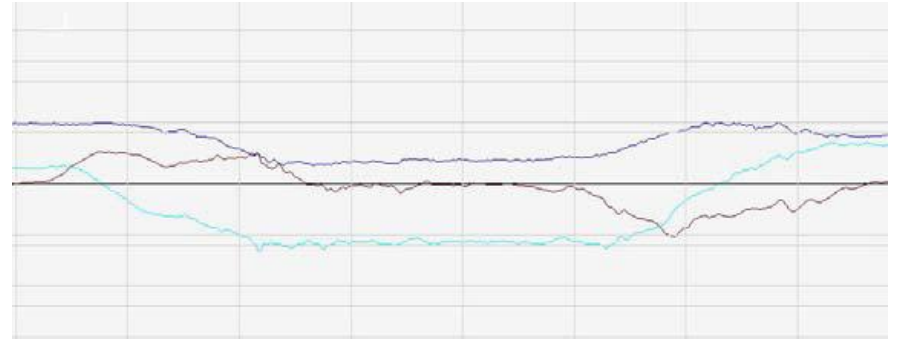

(a) "Lie down and then stand up slowly"

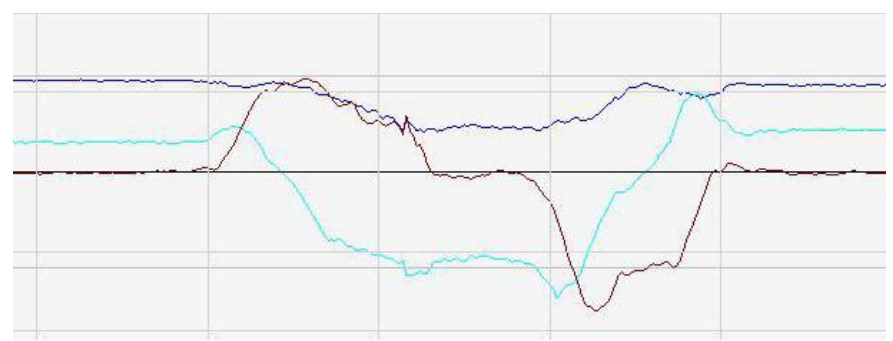

(b) "Lie down and then stand up quickly"

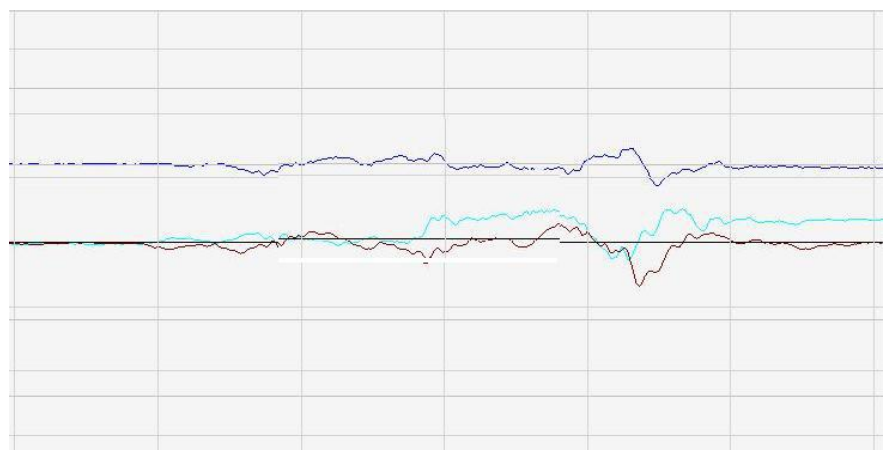

(c) "Sit down on the chair slowly"

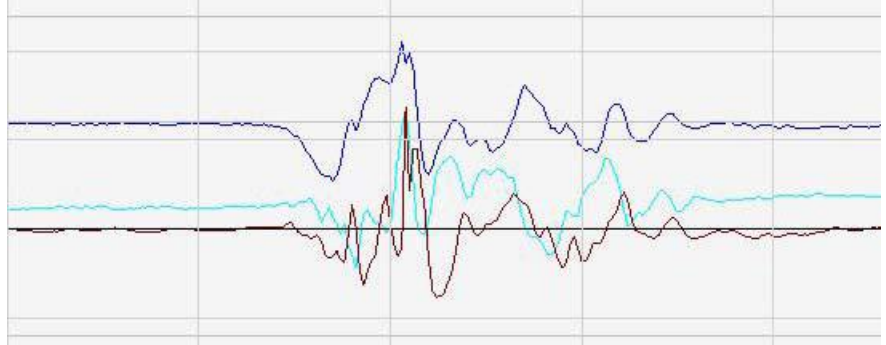

(d)"Sit down on the chair quickly"

Fig. 14. Validation of three axes of acceleration sensor and angular velocity sensor data (X: Dark Blue, Y: Blue, Z: Green for acceleration sensor, and X: Black, Y: Green, Z: Red for angular velocity sensor)

TABLE I. SUMMARY OF VALIDATION RESULTS FOR DETCTION OF USERS' ACTIONS OF "LIE DOWN AND THEN STAND UP" AND "SIT DOWN ON THE CHAIR"
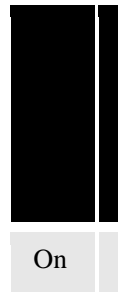

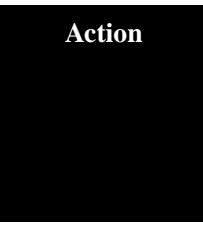

Accelerations $\mathrm{X}$

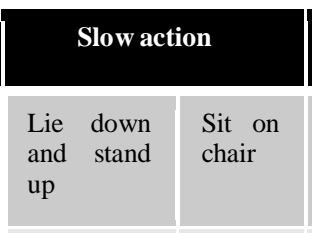

0

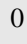

2

3

0

Accelerations Z

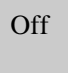

Off

On

Angular
Velocity Z

Angular
Velocity X

Angular Velocity Y Velocity Z

\begin{tabular}{|l|}
\hline \\
\hline 2 \\
\hline
\end{tabular}

1

3

\begin{tabular}{|l|}
\hline 2 \\
\hline 1 \\
\hline
\end{tabular}

1

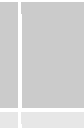

Quick action

\begin{tabular}{ll|l|} 
Lie down & $\begin{array}{l}\text { Sit on } \\
\text { chair } \\
\text { and stand } \\
\text { up }\end{array}$ & \\
\end{tabular}

2 3 2

\begin{tabular}{|l|l|l|}
\hline 0 & 2 & 1 \\
\hline 0 & 2 & 1 \\
\hline 1 & 3 & 2 \\
\hline
\end{tabular}

\section{CONCLUSION}

Method and system for human action detections with acceleration sensors for the proposed rescue system for disabled and elderly persons who need a help in evacuation from disaster areas is proposed. Not only vital signs, blood pressure, heart beat pulse rate, body temperature, bless and consciousness, but also, the location and attitude of the persons have to be monitored for the proposed rescue system. The attitude can be measured with acceleration sensors.

In particular, it is better to discriminate the attitudes, sitting, standing up, and lying down. Also, action speed has to be detected. Experimental results show that these attitude monitoring can be done with acceleration sensors.

Through these validations, it is found that the most effective signal for detection of lie down and then stand up is acceleration in $\mathrm{Y}$ axis while that for detecting of sit down action is angular velocity sensor data in $\mathrm{Z}$ axis. Namely, validation results for detection of users' actions of "Lie down and then stand up" and "Sit down on the chair"

\section{ACKNOWLEDGMENT}

The author would like to thank all the patients who are contributed to the experiments conducted. The author also would like to thank Professor Dr. Takao Hotokebuchi, President of Saga University for his support this research works.

\section{REFERENCES}

[1] Kohei Arai, Tran Xuan Sang, Decision making and emergency communication system in rescue simulation for people with disabilities, International Journal of Advanced Research in Artificial Intelligence, 2, 3, 77-85, 2013. 
[2] K.Arai, T.X.Sang, N.T.Uyen, Task allocation model for rescue disable persons in disaster area with help of volunteers, International Journal of Advanced Computer Science and Applications, 3, 7, 96-101, 2012.

[3] K.Arai, T.X.Sang, Emergency rescue simulation for disabled persons with help from volunteers, International Journal of Research and Review on Computer Science, 3, 2, 1543-1547, 2012.

[4] K. Arai, and T. X. Sang, "Fuzzy Genetic Algorithm for Prioritization Determination with Technique for Order Preference by Similarity to Ideal Solution", International Journal of Computer Science and Network Security, vol.11, no.5, 229-235, May 2011.

[5] Arai K., R. Mardiyanto, Evaluation of Students' Impact for Using the Proposed Eye Based HCI with Moving and Fixed Keyboard by Using EEG Signals, International Journal of Review and Research on Computer Science(IJRRCS), 2, 6, 1228-1234, 2011

[6] K.Arai, Wearable healthy monitoring sensor network and its application to evacuation and rescue information server system for disabled and elderly person, International Journal of Research and Review on Computer Science, 3, 3, 1633-1639, 2012.
[7] K.Arai, Wearable Physical and Psychological Health Monitoring System, Proceedings of the Science and Information Conference 2013 October 79, 2013 | London, UK

\section{Authors Profile}

Kohei Arai, He received BS, MS and PhD degrees in 1972, 1974 and 1982, respectively. He was with The Institute for Industrial Science and Technology of the University of Tokyo from April 1974 to December 1978 also was with National Space Development Agency of Japan from January, 1979 to March, 1990. During from 1985 to 1987, he was with Canada Centre for Remote Sensing as a Post Doctoral Fellow of National Science and Engineering Research Council of Canada. He moved to Saga University as a Professor in Department of Information Science on April 1990. He was a councilor for the Aeronautics and Space related to the Technology Committee of the Ministry of Science and Technology during from 1998 to 2000 . He was a councilor of Saga University for 2002 and 2003. He also was an executive councilor for the Remote Sensing Society of Japan for 2003 to 2005. He is an Adjunct Professor of University of Arizona, USA since 1998. He also is Vice Chairman of the Commission "A" of ICSU/COSPAR since 2008. He wrote 31 books and published 442 journal papers 